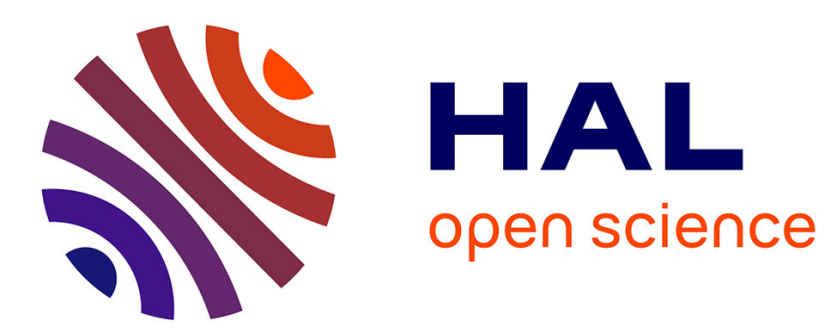

\title{
A Nitsche extended finite element method for incompressible elasticity with discontinuous modulus of elasticity
}

R. Becker, Erik Burman, Peter Hansbo

\section{- To cite this version:}

R. Becker, Erik Burman, Peter Hansbo. A Nitsche extended finite element method for incompressible elasticity with discontinuous modulus of elasticity. [Research Report] 2008. inria-00341737

\section{HAL Id: inria-00341737 \\ https://hal.inria.fr/inria-00341737}

Submitted on 25 Nov 2008

HAL is a multi-disciplinary open access archive for the deposit and dissemination of scientific research documents, whether they are published or not. The documents may come from teaching and research institutions in France or abroad, or from public or private research centers.
L'archive ouverte pluridisciplinaire HAL, est destinée au dépôt et à la diffusion de documents scientifiques de niveau recherche, publiés ou non, émanant des établissements d'enseignement et de recherche français ou étrangers, des laboratoires publics ou privés. 


\title{
A Nitsche extended finite element method for incompressible elasticity with discontinuous modulus of elasticity
}

\author{
Roland Becker $^{\mathrm{a}}$, Erik Burman ${ }^{\mathrm{b}}$ and Peter Hansbo ${ }^{\mathrm{c}}$ \\ ${ }^{a}$ Laboratoire de Mathématiques Appliques, Université de Pau et des Pays de \\ l'Adour, BP 1155, 64013 PAU Cedex, France \\ ${ }^{\mathrm{b}}$ Department of Mathematics, University of Sussex, Brighton, BN1 9RF United \\ Kingdom \\ ${ }^{\mathrm{c}}$ Department of Mathematical Sciences, Chalmers University of Technology and \\ University of Gothenburg, SE-412 96 Göteborg, Sweden
}

\begin{abstract}
In this note we propose a finite element method for incompressible (or compressible) elasticity problems with discontinuous modulus of elasticity (or, if compressible, Poisson's ratio). The problem is written on mixed form using $P^{1}$-continuous displacements and the space of piecewise $P^{0}$ pressures, leading to the possibility of eliminating the pressure beforehand in the compressible case. In the incompressible case, the method is augmented by a stabilization term, penalizing the pressure jumps. We show a priori error estimates under certain regularity hypothesis. In particular we prove that if the exact solution is sufficiently smooth in each subdomain then the convergence order is optimal.
\end{abstract}

\section{Introduction}

Hansbo and Hansbo $[5,6]$ proposed an unfitted finite element method for elliptic interface methods using Nitsche's method to allow for discontinuous or weakly discontinuous solutions. In particular, [6] deals with the elasticity problem, however avoiding the incompressible case. In this paper we aim to fill this gap.

We note that the equations of incompressible elasticity written on mixed form with the pressure as an auxiliary variable is form-identical to the Stokes equations of creeping viscous flow, and thus this paper serves as a basis for computations involving also two- (or multi-) fluid problems, which has been an 
important field of research during the last ten years. Techniques such as front tracking and front capturing have been introduced in order to keep track of the front. Both these techniques have qualities and drawbacks. Typically a front tracking algorithm will give a sharp resolution of the front and since the mesh follows the interface it is also well suited to solve the fluid mechanics equations with discontinuous coefficients that the two-fluid problem gives rise to; on the other hand it breaks down if topological changes occur in the exact solution. This is essentially the reason why front capturing method like volume of fluid or level set methods were introduced: they handle topological change automatically. However, since the position of the front is known only through intermediate of the interface capturing function the mesh is not fitted to the discontinuities of the coefficients in the exact solution. It is well known that for elliptic problems this leads to loss of convergence and pollution effects perturbing the solution close to the front. This paper thus addresses also the latter question in the setting of fluid mechanics.

We consider the space of piecewise affine, continuous, functions for the approximation of the velocities and the space of piecewise constant functions for the pressure. The pressure jumps between adjacent elements are penalized to enhance stability. Across the interface we enrich the finite element space so that both the velocities and the pressures are allowed to be discontinuous. The solutions in the two subdomains are then coupled using Nitsche's method.

We show that our formulation satisfies the inf-sup condition for stabilized methods related to the non-mixed constant strain method. This stability estimate is then used in combination with approximation estimates using suitable extension operators to prove prove optimal a priori estimates in energy and $L_{2}-$ norms.

\section{The elasticity problem on mixed form}

Let $\Omega$ be a bounded domain in $\mathbb{R}^{2}$, with convex polygonal boundary $\partial \Omega$ and an internal smooth boundary $\Gamma$, with bounded measure, dividing $\Omega$ into two open sets $\Omega_{1}$ and $\Omega_{2}$. The mixed form of the elasticity equations that we propose as a model problem is given by seeking the displacements $\boldsymbol{u}$ and the pressure $p$ such that

$$
\begin{aligned}
-\nabla \cdot\left(2 \mu_{i} \varepsilon(\boldsymbol{u})\right)+\nabla p & =f & & \text { in } \Omega_{i}, i=1,2, \\
\nabla \cdot \boldsymbol{u}-\frac{1}{\lambda_{i}} p & =0 & & \text { in } \Omega_{i}, i=1,2, \\
\boldsymbol{u} & =0 & & \text { on } \partial \Omega \backslash \Gamma .
\end{aligned}
$$


where, in terms of Young's modulus $E_{i}$ and Poisson's ratio $\nu_{i}$, we have $\mu_{i}=$ $E_{i} /\left(2\left(1+\nu_{i}\right)\right)$ and $\lambda_{i}=E_{i} \nu_{i} /\left(\left(1+\nu_{i}\right)\left(1-2 \nu_{i}\right)\right)$. (Strictly speaking, $p$ can only be identified as the pressure in the incompressible case $\nu_{i} \rightarrow 1 / 2$, but for simplicity we shall omit this distinction.) Here $\boldsymbol{\varepsilon}(\boldsymbol{u})$ is the symmetric part of the displacement gradient and $\Gamma$ denotes the interface between $\Omega_{1}$ and $\Omega_{2}, \Omega=\Omega_{1} \cup \Omega_{2}$ and the Lamé parameters $\mu_{i}$ and $\lambda_{i}$ are constant in each subdomain $\Omega_{i}$. For any sufficiently regular function $x$ in $\Omega_{1} \cup \Omega_{2}$ we define the jump of $x$ across $\Gamma$ by $\llbracket x \rrbracket=x_{1}-x_{2}$, where $x_{i}=\left.x\right|_{\Omega_{i}}$. We also define the limits in domain $\Omega_{1}$ or $\Omega_{2}$ on $\Gamma$ by $\left.p(\mathbf{x})\right|_{\Gamma^{ \pm}}=\lim _{\epsilon \rightarrow \pm 0} p(\mathbf{x}+\epsilon \boldsymbol{n})$ where $\mathbf{x} \in \Gamma$ and where $\boldsymbol{n}$ is the outward pointing normal to $\Omega_{1}$ on $\Gamma$. Let $(u, v)_{X}$ denote the $L^{2}$-scalar product on $X$,

$$
(u, v)_{X}:=\int_{X} u v \mathrm{~d} x
$$

with associated norm $\|u\|_{0, X}:=(u, u)_{X}^{\frac{1}{2}}$. The Sobolev-norms associated with the spaces $H^{k}(X)$ will be denoted by $\|u\|_{k, X}$. Define the the max and min of the shear moduli by $\mu_{\max }:=\max _{\Omega} \mu$ and $\mu_{\min }:=\min _{\Omega} \mu$. Formally we obtain the weak formulation by multiplying (1) by a function $(\boldsymbol{v}, q) \in\left[H_{0}^{1}(\Omega)\right]^{2} \times L^{2}(\Omega)=$ $V \times Q$ and integrating by parts, leading to the problem of finding $(\boldsymbol{u}, p) \in V \times Q$ such that

$$
\begin{aligned}
& \sum_{i=1}^{2}\left(\left(2 \mu_{i} \varepsilon(\boldsymbol{u}), \boldsymbol{\varepsilon}(\boldsymbol{v})\right)_{\Omega_{i}}-(p, \nabla \cdot \boldsymbol{v})_{\Omega_{i}}+(q, \nabla \cdot \boldsymbol{u})_{\Omega_{i}}-\left(\lambda_{i}^{-1} p, q\right)_{\Omega_{i}}\right) \\
& +\int_{\Gamma} \llbracket p \boldsymbol{n}-2 \mu \boldsymbol{\varepsilon}(\boldsymbol{u}) \cdot \boldsymbol{n} \rrbracket \cdot \boldsymbol{v} \mathrm{d} s=(\boldsymbol{f}, \boldsymbol{v})_{\Omega}, \text { for all }(\boldsymbol{v}, q) \in V \times Q
\end{aligned}
$$

where

$$
(\varepsilon(\boldsymbol{u}), \boldsymbol{\varepsilon}(\boldsymbol{v}))_{\Omega_{i}}:=\int_{\Omega_{i}} \boldsymbol{\varepsilon}(\boldsymbol{u}): \boldsymbol{\varepsilon}(\boldsymbol{v}) d x .
$$

Taking into account the following interface conditions

$$
\llbracket p \boldsymbol{n}-2 \mu \varepsilon(\boldsymbol{u}) \cdot \boldsymbol{n} \rrbracket \cdot \boldsymbol{n}=-\sigma \kappa
$$

and

$$
\llbracket 2 \mu \varepsilon(\boldsymbol{u}) \cdot \boldsymbol{n} \rrbracket \cdot \boldsymbol{t}=0,
$$

where $\boldsymbol{t}=\left(-n_{y}, n_{x}\right)$ and $\sigma \kappa$ denotes the surface tension force term, with $\kappa$ the interface curvature, we arrive at the following well posed formulation: Find $(u, p) \in V \times Q$ such that

$$
B[(\boldsymbol{u}, p),(\boldsymbol{v}, q)]:=(\boldsymbol{f}, \boldsymbol{v})_{\Omega}+\int_{\Gamma} \sigma \kappa \boldsymbol{v} \cdot \boldsymbol{n} \mathrm{d} s, \text { for all }(\boldsymbol{v}, q) \in V \times Q,
$$

where

$$
\begin{aligned}
B[(\boldsymbol{u}, p),(\boldsymbol{v}, q)]=\sum_{i=1}^{2}( & \left(2 \mu_{i} \boldsymbol{\varepsilon}(\boldsymbol{u}), \boldsymbol{\varepsilon}(\boldsymbol{v})\right)_{\Omega_{i}}-(p, \nabla \cdot \boldsymbol{v})_{\Omega_{i}} \\
& \left.+(q, \nabla \cdot \boldsymbol{u})_{\Omega_{i}}-\left(\lambda_{i}^{-1} p, q\right)_{\Omega_{i}}\right) .
\end{aligned}
$$


For the analysis we will only consider the incompressible case to reduce the technical detail. When both materials are incompressible the pressure space must be chosen as $L_{0}^{2}(\Omega)$. The extension to the compressible case is straightforward combining the results below with those of [6] Moreover for the convergence analysis we will assume that $\boldsymbol{u}$ is in $H^{2}$ on each subdomain and $p$ in $H^{1}$ in each subdomain. For a regularity analysis in the compressible case we refer to [8] and in the incompressible case to [9].

\section{The finite element formulation}

In a standard finite element method the jump in normal derivative resulting from the continuity of the traction, when $\mu_{1} \neq \mu_{2}$ or $\lambda_{1} \neq \lambda_{2}$, can be taken into account by letting $\Gamma$ coincide with meshlines. However, for optimization or inverse identification purposes (or in general for two-fluid problems) where the interface will have to move, this implies remeshing during the simulation, at least close to the interface. Instead we propose to solve (1) approximately using piecewise linear displacements and piecewise constant pressures on a family of conforming triangulations $\mathcal{T}_{h}$ of $\Omega$ which are independent of the location of the interface. To avoid loss in convergence order we relax the continuity requirement over $\Gamma$ and allow the approximation to be discontinuous inside elements which intersect the interface.

We will use the following notation for mesh related quantities. Let $h_{K}$ be the diameter of $K$ and $h=\max _{K \in \mathcal{T}_{h}} h_{K}$. For any element $K$, let $K_{i}=K \cap \Omega_{i}$ denote the part of $K$ in $\Omega_{i}$. By $G_{h}:=\left\{K \in \mathcal{T}_{h}: K \cap \Gamma \neq \emptyset\right\}$ we denote the set of elements that are intersected by the interface. For an element $K \in G_{h}$, let $\Gamma_{K}:=\Gamma \cap K$ be the part of $\Gamma$ intersecting $K$.

We make the following assumptions regarding the mesh and the interface (from $[5])$.

- A1: We assume that the triangulation is non-degenerate, i.e.,

$$
h_{K} / \rho_{K} \leq C \quad \forall K \in \mathcal{T}_{h}
$$

where $h_{K}$ is the diameter of $K$ and $\rho_{K}$ is the diameter of the largest ball contained in $K$.

- A2: We assume that $\Gamma$ intersects each element boundary $\partial K$ exactly twice and each (open) edge at most once.

- A3: Let $\Gamma_{K, h}$ be the straight line segment connecting the points of intersection between $\Gamma$ and $\partial K$. We assume that $\Gamma_{K}$ is a function of length on $\Gamma_{K, h}$; in local coordinates

$$
\Gamma_{K, h}=\left\{(\xi, \eta): 0<\xi<\left|\Gamma_{K, h}\right|, \eta=0\right\}
$$


and

$$
\Gamma_{K}=\left\{(\xi, \eta): 0<\xi<\left|\Gamma_{K, h}\right|, \eta=\delta(\xi)\right\}
$$

Since the curvature of $\Gamma$ is bounded the assumptions A2 and A3 are always fulfilled on sufficiently fine meshes. These assumptions essentially demand that the interface is well resolved by the mesh.

To obtain the finite element formulation corresponding to (5) we replace the test function $\boldsymbol{v}$ by $\tilde{\boldsymbol{v}}$ such that $\left.\tilde{\boldsymbol{v}}\right|_{\Omega_{i}}=\boldsymbol{v}_{i}$ with $\boldsymbol{v}_{i} \in\left[H^{1}\left(\Omega_{i}\right)\right]^{2}$ and $\boldsymbol{v}_{i}=0$ on the boundary $\partial \Omega$ thus allowing for a discontinuity over the interface $\Gamma$.

For the average across $\Gamma$ of any sufficiently smooth function $x$ discontinuous across $\Gamma$ we will use the notation $\{x\}=\kappa_{1} x_{1}+\kappa_{2} x_{2}$ and $\langle x\rangle=\kappa_{2} x_{1}+\kappa_{1} x_{2}$ with $\left.\kappa_{i}\right|_{\Gamma_{K}}=\left|K_{i}\right| /|K|$. Note that $\kappa_{1}+\kappa_{2}=1$. We have

$$
x_{1}=\{x\}+\kappa_{2} \llbracket x \rrbracket \text { and } x_{2}=\{x\}-\kappa_{1} \llbracket x \rrbracket,
$$

and therefore

$$
\llbracket a b \rrbracket=\{a\} \llbracket b \rrbracket+\llbracket a \rrbracket\langle b\rangle .
$$

We note that since $\nabla \cdot(p \mathcal{I}-2 \mu \boldsymbol{\varepsilon}(\boldsymbol{u})) \in L^{2}(\Omega)$ we have $(p \boldsymbol{n}-2 \mu \boldsymbol{\varepsilon}(\boldsymbol{u}) \cdot \boldsymbol{n}) \in$ $H^{-\frac{1}{2}}(\Gamma)$. Integrating by parts and using $(7)$ we now obtain for the interface term

$$
\begin{aligned}
& \int_{\Gamma} \llbracket(p \boldsymbol{n}-2 \mu \boldsymbol{\varepsilon}(\boldsymbol{u}) \cdot \boldsymbol{n}) \tilde{\boldsymbol{v}} \rrbracket \mathrm{d} s= \\
& \int_{\Gamma}\{p \boldsymbol{n}-2 \mu \boldsymbol{\varepsilon}(\boldsymbol{u}) \cdot \boldsymbol{n}\} \llbracket \tilde{\boldsymbol{v}} \rrbracket \mathrm{d} s+\int_{\Gamma} \llbracket p \boldsymbol{n}-2 \mu \varepsilon(\boldsymbol{u}) \cdot \boldsymbol{n} \rrbracket\langle\tilde{\boldsymbol{v}}\rangle \mathrm{d} s .
\end{aligned}
$$

Since $\tilde{\boldsymbol{v}} \in\left[H^{1}(\Omega)\right]^{2}$ the quantities in (8) are well defined if the interface integrals are interpreted as duality pairings $H^{-\frac{1}{2}}(\Gamma) / H^{\frac{1}{2}}(\Gamma)$.

Using now the interface conditions (3) and (4), with $\kappa=0$, in the second term we have

$$
\int_{\Gamma} \llbracket p \boldsymbol{n}-2 \mu \varepsilon(\boldsymbol{u}) \cdot \boldsymbol{n} \rrbracket\langle\tilde{\boldsymbol{v}}\rangle \mathrm{d} s=-\int_{\Gamma} \sigma \kappa\langle\tilde{\boldsymbol{v}} \cdot \boldsymbol{n}\rangle \mathrm{d} s .
$$

After these preliminary considerations we are now ready to propose a finite element discretization of the problem, with this aim we introduce two conforming triangulations $\mathcal{T}_{1}$ and $\mathcal{T}_{2}$ such that the union of $\mathcal{T}_{1}$ and $\mathcal{T}_{2}$ gives a conforming triangulation of all $\Omega$ and for every triangle $K \in \mathcal{T}_{1} \cap \mathcal{T}_{2}$ we have $K \cap \Gamma \neq \emptyset$. We also denote the set of interior faces of $\mathcal{T}_{i}$ by $\mathcal{F}_{i}$. Associated with $\mathcal{T}_{1}$ and $\mathcal{T}_{2}$ we have the finite element spaces

$$
\begin{gathered}
V_{i}^{h}=\left\{\boldsymbol{v} \in\left[C^{0}\left(\Omega_{i}\right)\right]^{2}:\left.\boldsymbol{v}\right|_{K} \in\left[P^{1}(K)\right]^{2}, \forall K \in \mathcal{T}_{i},\left.\boldsymbol{v}\right|_{\partial \Omega}=0\right\}, \\
Q_{i}^{h}=\left\{v \in L_{0}^{2}\left(\Omega_{i}\right):\left.v\right|_{K} \in P^{0}(K), \forall K \in \mathcal{T}_{i}\right\} .
\end{gathered}
$$

Moreover we denote $V_{h}=V_{1}^{h} \times V_{2}^{h}$ and $Q_{h}=Q_{1}^{h} \times Q_{2}^{h}$. The finite element discretisation now takes the form: find $\left(\boldsymbol{u}_{i, h}, p_{i, h}\right) \in V_{i}^{h} \times Q_{i}^{h}, i=1,2$ such 
that $\boldsymbol{u}_{h}=\left(\boldsymbol{u}_{1, h}, \boldsymbol{u}_{2, h}\right), p_{h}=\left(p_{1, h}, p_{2, h}\right)$ satisfy

$$
B_{h}\left[\left(\boldsymbol{u}_{h}, p_{h}\right),\left(\boldsymbol{v}_{h}, q_{h}\right)\right]+J\left(p_{h}, q_{h}\right)=\left(\boldsymbol{f}, \boldsymbol{v}_{h}\right)+\int_{\Gamma} \sigma \kappa\left\langle\boldsymbol{v}_{h} \cdot \boldsymbol{n}\right\rangle \mathrm{d} s,
$$

for all $\left(\boldsymbol{v}_{h}, q_{h}\right) \in V_{h} \times Q_{h}$, where

$$
\begin{gathered}
B_{h}\left[\left(\boldsymbol{u}_{h}, p_{h}\right),\left(\boldsymbol{v}_{h}, q_{h}\right)\right]=a_{h}\left(\boldsymbol{u}_{h}, \boldsymbol{v}_{h}\right)+b_{h}\left(p_{h}, \boldsymbol{v}_{h}\right)-b_{h}\left(q_{h}, \boldsymbol{u}_{h}\right) \\
a_{h}\left(\boldsymbol{u}_{h}, \boldsymbol{v}_{h}\right)=\int_{\Omega} 2 \mu \varepsilon\left(\boldsymbol{u}_{h}\right): \boldsymbol{\varepsilon}\left(\boldsymbol{v}_{h}\right) \mathrm{d} x-\int_{\Gamma}\left\{2 \mu \varepsilon\left(\boldsymbol{u}_{h}\right) \cdot \boldsymbol{n}\right\} \llbracket \boldsymbol{v}_{h} \rrbracket \mathrm{d} s \\
-\int_{\Gamma}\left\{2 \mu \varepsilon\left(\boldsymbol{v}_{h}\right) \cdot \boldsymbol{n}\right\} \llbracket \boldsymbol{u}_{h} \rrbracket \mathrm{d} s+\int_{\Gamma} \gamma_{u} \mu_{\max } h^{-1} \llbracket \boldsymbol{u}_{h} \rrbracket \llbracket \boldsymbol{v}_{h} \rrbracket \mathrm{d} s, \\
b_{h}\left(p_{h}, v_{h}\right)=-\int_{\Omega} p_{h} \nabla \cdot \boldsymbol{v}_{h} \mathrm{~d} x+\int_{\Gamma}\left\{p_{h}\right\} \llbracket \boldsymbol{v}_{h} \cdot \boldsymbol{n} \rrbracket \mathrm{d} s,
\end{gathered}
$$

and

$$
J\left(p_{h}, q_{h}\right)=\sum_{F \in \mathcal{F}_{1}} \int_{F} \frac{\gamma_{1}}{\mu_{1}} h_{K}\left[p_{1, h}\right]\left[q_{1, h}\right] \mathrm{d} s+\sum_{F \in \mathcal{F}_{2}} \int_{F} \frac{\gamma_{2}}{\mu_{2}} h_{K}\left[p_{2, h}\right]\left[q_{2, h}\right] \mathrm{d} s .
$$

where $[p]$ denotes the jump of $p$ across an element face $F$ and $\left(\gamma_{u}, \gamma_{1}, \gamma_{2}\right)$ are constants, the size of which will be discussed in the following.

Remark 1 Note that the velocities and the pressures are double valued in the overlap region. Moreover the pressure from both sides are stabilized over the whole interior face in the overlapping domain.

The formulation (10) satisfies the following consistency relation

Lemma 2 (Galerkin orthogonality) Let $\left(\boldsymbol{u}_{h}, p_{h}\right)$ be the solution of the finite element formulation (10) and $(\boldsymbol{u}, p) \in\left[H^{2}\left(\Omega_{1}\right) \cup H^{2}\left(\Omega_{2}\right)\right]^{2} \times H^{1}\left(\Omega_{1}\right) \cup H^{1}\left(\Omega_{2}\right)$ be the solution of (5) then

$$
B_{h}\left[\left(\boldsymbol{u}_{h}-\boldsymbol{u}, p_{h}-p\right),\left(\boldsymbol{v}_{h}, q_{h}\right)\right]=-J\left(p_{h}, q_{h}\right) \quad \forall\left(\boldsymbol{v}_{h}, q_{h}\right) \in V_{h} \times Q_{h} .
$$

PROOF. Using the formulation (10) we may write

$$
a_{h}\left(\boldsymbol{u}, \boldsymbol{v}_{h}\right)+b_{h}\left(p, \boldsymbol{v}_{h}\right)-b_{h}\left(q_{h}, \boldsymbol{u}\right)=\left(\boldsymbol{f}, \boldsymbol{v}_{h}\right)+\int_{\Gamma} \sigma \kappa\left\langle v_{h} \cdot \boldsymbol{n}\right\rangle \mathrm{d} s .
$$

The claim follows by noting that $b_{h}\left(q_{h}, \boldsymbol{u}\right)=0$ and by integrating by parts in the remaining two terms on the left hand side and using equality (9) and equation (1).

A FE basis for $V_{h}$ is easily obtained from a standard FE basis on the mesh by the introduction of new basis functions for the elements that intersect $\Gamma$. 
For piecewise linears, the standard interior nodal basis functions in $\Omega$ may be partitioned into the two sets $\left\{\psi_{i}^{j}\right\}_{j=1}^{N_{i}}$ of basis functions with support entirely in $\Omega_{i}, i=1,2$, and the set $\left\{\psi_{\Gamma}^{k}\right\}_{k=1}^{M}$ of basis functions which are non-zero on $\Gamma$. For each of the latter, let $\psi_{\Gamma, i}^{j}:=\left.\psi_{\Gamma}^{j}\right|_{\Omega_{i}}$. Then $\left\{\psi_{i}^{j}\right\}_{j=1}^{N_{i}} \cup\left\{\psi_{\Gamma, i}^{k}\right\}_{k=1}^{M}$ is a basis for $V_{i}^{h}$. As a consequence, the number of basis functions on each element that intersects $\Gamma$ is doubled.

\section{Approximation properties}

The solution of (1) looses regularity at the interface. Indeed the pressure and normal component of the deformation tensor may jump. We introduce the broken Sobolev spaces

$$
H^{k}\left(\Omega_{1} \cup \Omega_{2}\right):=\left\{v:\left.v\right|_{\Omega_{i}} \in H^{k}\left(\Omega_{i}\right), i=1,2\right\}
$$

with the corresponding norms

$$
\|u\|_{k, \Omega_{1} \cup \Omega_{2}}^{2}:=\sum_{i=1}^{2}\|u\|_{k, \Omega_{i}}^{2}
$$

We need to show that our approximating spaces $V_{h}$ and $Q_{h}$ have optimal approximation properties on $H^{2}\left(\Omega_{1} \cup \Omega_{2}\right)$ and $H^{1}\left(\Omega_{1} \cup \Omega_{2}\right)$ respectively. This follows from some minor modifications of the analysis in [5]. Let $G_{h}$ denote the set of elements that are intersected by the interface. We will use the following mesh dependent norms defined for functions in $\left[H^{2}\left(\Omega_{1} \cup \Omega_{2}\right)\right]^{2}$ or $\left[H^{2}\left(\Omega_{1} \cup \Omega_{2}\right)\right]^{2} \times H^{1}(\Omega)$ :

$$
\begin{aligned}
\|\boldsymbol{v}\|_{1 / 2, h, \Gamma}^{2} & :=\sum_{K \in G_{h}} h_{K}^{-1}\|\boldsymbol{v}\|_{0, \Gamma_{K}}^{2}, \\
\|\boldsymbol{v}\|_{-1 / 2, h, \Gamma}^{2} & :=\sum_{K \in G_{h}} h_{K}^{1}\|\boldsymbol{v}\|_{0, \Gamma_{K}}^{2},
\end{aligned}
$$

and

$$
\|\boldsymbol{v}\|\left\|^{2}:=\right\| \boldsymbol{v}\left\|_{0, \Omega}^{2}+\right\| \mu^{1 / 2} \nabla \boldsymbol{v}\left\|_{0, \Omega}^{2}+\right\|\{2 \mu \boldsymbol{\varepsilon}(\boldsymbol{v}) \cdot \boldsymbol{n}\}\left\|_{-1 / 2, h, \Gamma}^{2}+\right\|[\boldsymbol{v}] \|_{1 / 2, h, \Gamma}^{2} .
$$

On the product space $V \times Q$ we introduce the norm

$$
\left\|\left(\boldsymbol{u}_{h}, p_{h}\right)\right\|^{2}:=\left\|\boldsymbol{u}_{h}\right\|^{2}+\frac{1}{\mu_{\max }}\left\|p_{h}\right\|_{0, \Omega}^{2}
$$

and on $V_{h} \times Q_{h}$ the norm

$$
\left\|\left(\boldsymbol{u}_{h}, p_{h}\right)\right\|_{h}^{2}:=\left\|\boldsymbol{u}_{h}\right\|^{2}+\frac{1}{\mu_{\max }}\left\|p_{h}\right\|_{0, \Omega}^{2}+J\left(p_{h}, p_{h}\right) .
$$


We note for future reference that

$$
(\boldsymbol{u}, \boldsymbol{v})_{\Gamma} \leq\|\boldsymbol{u}\|_{1 / 2, h, \Gamma}\|\boldsymbol{v}\|_{-1 / 2, h, \Gamma} .
$$

To show that functions in the space $\left(V_{h}, Q_{h}\right)$ approximate functions $\boldsymbol{v} \in$ $\left[H_{0}^{1}(\Omega) \cap H^{2}\left(\Omega_{1} \cup \Omega_{2}\right)\right]^{2}$ and $q \in L_{0}^{2}(\Omega) \cap H^{1}\left(\Omega_{1} \cup \Omega_{2}\right)$ to the order $h$ in the norm $\|\cdot \mid\|$ and $\|\cdot\|_{0, \Omega}$, respectively, we construct an interpolant of $(\boldsymbol{v}, q)$ by standard interpolants of $\left[H^{2}\right]^{2} \times H^{1}$-extensions of $\left(\boldsymbol{v}_{1}, q_{1}\right)$ and $\left(\boldsymbol{v}_{2}, q_{2}\right)$ as follows. Choose extension operators $\mathrm{E}_{i}^{k}: H^{k}\left(\Omega_{i}\right) \rightarrow H^{k}(\Omega)$ such that $\left.\left(\mathrm{E}_{i}^{2} \boldsymbol{v}, \mathrm{E}_{i}^{1} q\right)\right|_{\Omega_{i}}=(\boldsymbol{v}, q)$ and

$$
\left\|\mathrm{E}_{i}^{k} w\right\|_{s, \Omega} \leq C\|w\|_{s, \Omega_{i}}, \quad \forall w \in H^{s}\left(\Omega_{i}\right), s=0, \ldots, k .
$$

Let $I_{h}$ be the standard interpolant and $C_{h}$ be the local projection on the piecewise constants. We define

$$
\left(I_{h}^{*} \boldsymbol{v}, C_{h}^{*} q\right):=\left(\left(I_{h, 1}^{*} \boldsymbol{v}_{1}, I_{h, 2}^{*} \boldsymbol{v}_{2}\right),\left(C_{h, 1}^{*} q_{1}, C_{h, 2}^{*} q_{2}\right)\right),
$$

where $I_{h, i}^{*} \boldsymbol{v}_{i}:=\left.\left(I_{h} \mathrm{E}_{i}^{2} \boldsymbol{v}_{i}\right)\right|_{\Omega_{i}}$ and $C_{h, i}^{*} q_{i}:=\left.\left(C_{h} \mathrm{E}_{i}^{1} q_{i}\right)\right|_{\Omega_{i}}$. The following theorem is valid.

Theorem 3 Let $\left(I_{h}^{*}, C_{h}^{*}\right)$ be a pair of interpolation operators defined as in (15). Then

$$
\left\|\boldsymbol{v}-I_{h}^{*} \boldsymbol{v}\right\| \mid \leq C h\|\boldsymbol{v}\|_{2, \Omega_{1} \cup \Omega_{2}}
$$

and

$$
\left\|q-C_{h}^{*} q\right\|_{0, \Omega} \leq C h\|q\|_{1, \Omega_{1} \cup \Omega_{2}}
$$

for all $\boldsymbol{v} \in\left[H_{0}^{1}(\Omega) \cap H^{2}\left(\Omega_{1} \cup \Omega_{2}\right)\right]^{2}$ and $p \in L_{0}^{2}(\Omega) \cap H^{1}\left(\Omega_{1} \cup \Omega_{2}\right)$.

For the proof of this we need the following variant of a trace inequality on a reference element that we recall from [5] and state here without proof.

Lemma 4 Map a triangle $K \in G_{h}$ onto the unit reference triangle $\tilde{K}$ by an affine map and denote by $\tilde{\Gamma}_{\tilde{K}}$ the corresponding image of $\Gamma_{K}$. Under the the assumptions A1-A3 of Section 3 there exists a constant $C$, depending on $\Gamma$ but independent of the mesh, such that

$$
\|w\|_{0, \tilde{\Gamma}_{\tilde{K}}}^{2} \leq C\|w\|_{0, \tilde{K}}\|w\|_{1, \tilde{K}}, \quad \forall w \in H^{1}(\tilde{K}) .
$$

PROOF. (Theorem 3)

Recall that $K_{i}=K \cap \Omega_{i}$ and let $\boldsymbol{v}_{i}^{*}=\mathrm{E}_{i}^{2} \boldsymbol{v}_{i}$ denote the extension of $\boldsymbol{v}_{i}$ to $\Omega$ and similarly $q_{i}^{*}=\mathrm{E}_{i}^{1} q_{i}$ denote the extension of $q_{i}$ to $\Omega$. By standard interpolation estimates for $I_{h}$ and $C_{h}$ respectively we now obtain

$$
\begin{aligned}
\left\|\nabla\left(\boldsymbol{v}_{i}-I_{h, i}^{*} \boldsymbol{v}_{i}\right)\right\|_{0, K_{i}}^{2} & =\left\|\nabla\left(\boldsymbol{v}_{i}^{*}-I_{h, i}^{*} \boldsymbol{v}_{i}^{*}\right)\right\|_{0, K_{i}}^{2} \leq\left\|\nabla\left(\boldsymbol{v}_{i}^{*}-I_{h, i}^{*} \boldsymbol{v}_{i}^{*}\right)\right\|_{0, K}^{2} \\
& \leq C h_{K}^{2}\left\|\boldsymbol{v}_{i}^{*}\right\|_{2, K}^{2}
\end{aligned}
$$


and in the same fashion

$$
\left\|q_{i}-C_{h, i}^{*} q_{i}\right\|_{0, K_{i}}^{2} \leq C h_{K}^{2}\left\|q_{i}^{*}\right\|_{1, K}^{2}
$$

Summing over all triangles we obtain using (14)

$$
\left\|\nabla\left(\boldsymbol{v}_{i}-I_{h, i}^{*} \boldsymbol{v}_{i}\right)\right\|_{0, \Omega_{i}}^{2} \leq C h^{2} \sum_{K \cap \Omega_{i} \neq \emptyset}\left\|\boldsymbol{v}_{i}^{*}\right\|_{2, K}^{2} \leq C h^{2}\left\|\boldsymbol{v}_{i}\right\|_{2, \Omega_{i}}^{2}
$$

and

$$
\left\|q_{i}-C_{h, i}^{*} q_{i}\right\|_{0, \Omega_{i}}^{2} \leq C h^{2}\left\|q_{i}\right\|_{1, \Omega_{i}}^{2} .
$$

We turn now to the jumps on the interface. Since the mesh is non-degenerate, it follows from Lemma 4, scaled by the map from the reference triangle, that for $s \in \mathbb{R}$

$$
h_{K}^{-s}\|w\|_{0, \Gamma_{K}}^{2} \leq C\left(h_{K}^{-1-s}\|w\|_{0, K}^{2}+h_{K}^{1-s}\|w\|_{1, K}^{2}\right), \quad \forall w \in H^{1}(K)
$$

for $s \in \mathbb{R}$. Hence it follows, using once again standard interpolation estimates, that

$$
\begin{aligned}
h_{K}^{-1}\left\|\llbracket \boldsymbol{v}-I_{h}^{*} \boldsymbol{v} \rrbracket\right\|_{0, \Gamma_{K}}^{2} & \leq C h_{K}^{-1} \sum_{i}\left\|\boldsymbol{v}_{i}-I_{h, i}^{*} \boldsymbol{v}_{i}\right\|_{0, \Gamma_{K}}^{2} \\
& =C h_{K}^{-1} \sum_{i}\left\|\boldsymbol{v}_{i}^{*}-I_{h} \boldsymbol{v}_{i}^{*}\right\|_{0, \Gamma_{K}}^{2} \\
& \leq C \sum_{i}\left(h_{K}^{-2}\left\|\boldsymbol{v}_{i}^{*}-I_{h} \boldsymbol{v}_{i}^{*}\right\|_{0, K}^{2}+\left\|\boldsymbol{v}_{i}^{*}-I_{h} \boldsymbol{v}_{i}^{*}\right\|_{1, K}^{2}\right) \\
& \leq C h_{K}^{2} \sum_{i}\left\|\boldsymbol{v}_{i}^{*}\right\|_{2, K}^{2}
\end{aligned}
$$

and Summing the contributions from $K \in G_{h}$, we get from (14) that

$$
\begin{gathered}
\left\|\llbracket \boldsymbol{v}-I_{h}^{*} \boldsymbol{v} \rrbracket\right\|_{1 / 2, h, \Gamma} \leq C h \sum_{i}\left\|\boldsymbol{v}_{i}^{*}\right\|_{2, \bigcup K \in G_{h}} \leq C h\|\boldsymbol{v}\|_{2, \Omega_{1} \cup \Omega_{2}} \quad \text { and } \\
\left\|\llbracket q-C_{h}^{*} q \rrbracket\right\|_{-1 / 2, h, \Gamma} \leq C h \sum_{i}\left\|q_{i}^{*}\right\|_{1, \bigcup K \in G_{h}} \leq C h\|q\|_{1, \Omega_{1} \cup \Omega_{2}} .
\end{gathered}
$$

Next, we consider the mean viscous stress at the interface. Lemma 4 applied to $\boldsymbol{n} \cdot \nabla \boldsymbol{w}$ and scaling gives

$$
h_{K}\|\boldsymbol{n} \cdot \nabla \boldsymbol{w}\|_{0, \Gamma_{K}}^{2} \leq C\left(\|\boldsymbol{w}\|_{1, K}^{2}+h_{K}^{2}\|\boldsymbol{w}\|_{2, K}^{2}\right), \quad \forall \boldsymbol{w} \in\left[H^{2}(K)\right]^{2}
$$


Using this result applied to $\boldsymbol{w}=\boldsymbol{v}_{i}^{*}-I_{h} \boldsymbol{v}_{i}^{*}$ and again standard interpolation estimates, it follows that

$$
\begin{aligned}
h_{K}\left\|\left\{\boldsymbol{n} \cdot \nabla\left(\boldsymbol{v}-I_{h}^{*} \boldsymbol{v}\right)\right\}\right\|_{0, \Gamma_{K}}^{2} & \leq C h_{K} \sum_{i}\left\|\nabla\left(\boldsymbol{v}_{i}-I_{h, i}^{*} \boldsymbol{v}_{i}\right) \cdot \boldsymbol{n}\right\|_{0, \Gamma_{K}}^{2} \\
& =C h_{K} \sum_{i}\left\|\nabla\left(\boldsymbol{v}_{i}^{*}-I_{h} \boldsymbol{v}_{i}^{*}\right) \cdot \boldsymbol{n}\right\|_{0, \Gamma_{K}}^{2} \\
& \leq C \sum_{i}\left(h_{K}\left\|\boldsymbol{v}_{i}^{*}-I_{h} \boldsymbol{v}_{i}^{*}\right\|_{1, K}^{2}+h_{K}^{2}\left\|\boldsymbol{v}_{i}^{*}-I_{h} \boldsymbol{v}_{i}^{*}\right\|_{2, K}^{2}\right) \\
& \leq C h_{K}^{2} \sum_{i}\left\|\boldsymbol{v}_{i}^{*}\right\|_{2, K}^{2} .
\end{aligned}
$$

Summing again the contributions from $K \in G_{h}$, we from (14) that

$$
\left\|\left\{\boldsymbol{n} \cdot \nabla\left(\boldsymbol{v}_{i}-I_{h, i}^{*} \boldsymbol{v}_{i}\right)\right\}\right\|_{-1 / 2, h, \Gamma} \leq C h\left\|\boldsymbol{v}_{i}\right\|_{2, \Omega_{i}} .
$$

The claim now follows from equations (17), (18), (19).

Lemma 5 Let $q \in H^{1}\left(\Omega_{1} \cup \Omega_{2}\right)$ then there holds

$$
J\left(C_{h}^{*} q^{*}, C_{h}^{*} q^{*}\right) \leq C h^{2}\|q\|_{H^{1}\left(\Omega_{1} \cup \Omega_{2}\right)}^{2} .
$$

PROOF. This is easily proved using the standard trace inequality in the following fashion

$$
\begin{aligned}
h_{K}\left\|\left[q_{i}^{*}-C_{h}^{*} q_{i}^{*}\right]\right\|_{0, F}^{2} & \leq C\left(\left\|q_{i}^{*}-C_{h}^{*} q_{i}^{*}\right\|_{0, K}^{2}+h_{K}^{2}\left\|q_{i}^{*}\right\|_{1, K}^{2}\right) \\
& \leq C h_{K}^{2} \sum\left\|q_{i}^{*}\right\|_{1, K}^{2} .
\end{aligned}
$$

This leads to

$$
\begin{aligned}
J\left(C_{h}^{*} q^{*}, C_{h}^{*} q^{*}\right) & \leq \sum_{K \in \mathcal{T}_{1}} C h_{K}^{2} \sum\left\|q_{1}^{*}\right\|_{1, K}^{2}+\sum_{K \in \mathcal{T}_{2}} C h_{K}^{2} \sum\left\|q_{2}^{*}\right\|_{1, K}^{2} \\
& \leq C h^{2}\left(\|q\|_{1, \Omega_{1}}^{2}+\|q\|_{1, \Omega_{2}}^{2}\right)
\end{aligned}
$$

\section{The inf-sup condition}

We need to show that the formulation satisfies an inf-sup condition with regard to the norm $\|(\cdot, \cdot)\|_{h}$ in the case $\lambda_{i} \rightarrow \infty$. We remark that if $\lambda_{i}$ remain bounded from above, the pressure does not need stabilization and can be 
eliminated elementwise, recovering the constant-strain finite element method. In this case there is no stability problem in the pressure, and the results of [6] can be directly applied in the compressible subdomain. We shall therefore in the following concentrate on the case when both materials are assumed incompressible.

Observe that the pressure stabilization in $J(\cdot, \cdot)$ acts over the whole interior faces in $G_{h}$, i.e. not only the part of the faces in the computational domain $\Omega$. This is of fundamental importance for the below analysis, indeed this "ghost penalty" is what gives us stability of the interface pressure.

Here we prove the inf-sup condition only in the particular case of the mixed stabilized $P_{1} \times P_{0}$ formulation for convenience. However, the general framework holds also when using for instance the pressure stabilisations proposed in [4] or [1] and we expect the inf-sup condition to hold uniformly in these cases as well, provided the stabilization operator acts in all elements of $\mathcal{T}_{1}$ and $\mathcal{T}_{2}$.

We need the following inverse inequality, for a proof of which we refer to [5].

Lemma 6 For $v \in V_{h}$, the following inverse inequality holds:

$$
\|\{\boldsymbol{n} \cdot \nabla \boldsymbol{v}\}\|_{-1 / 2, h, \Gamma}^{2} \leq C_{I}\|\nabla \boldsymbol{v}\|_{0, \Omega_{1} \cup \Omega_{2}}^{2}
$$

\subsection{Fortin interpolant}

The key result for the inf-sup condition is the construction of the Fortin interpolant. The upshot in the case of Nitsche XFEM for Stokes' problem is the need to construct a Fortin interpolant with some additional orthogonality on the interface. The interpolant defined on mesh $\mathcal{T}_{i}$ will be denoted by $\pi_{h}^{i}$ and the global interpolant by $\pi_{h}$. Note that the global interpolant is not continuous across the interface. Let us consider the construction of the Fortin interpolant on the space $V_{1}^{h}$ the construction on $V_{2}^{h}$ then follows by symmetry. Let $\mathcal{T}_{\Gamma}$ by the subset of triangles of $\mathcal{T}_{1}$ such that

$$
\mathcal{T}_{\Gamma}=\left\{T \in \mathcal{T}_{1}: T \cap\left(\mathcal{T}_{1} \cap \mathcal{T}_{2}\right) \neq \emptyset\right\} .
$$

That is all the elements cut by the interface and the elements sharing a face or a vertex with them. This is a subset of the mesh of width approximately $2 h$. Let us now regroup the elements in $\mathcal{T}_{\Gamma}$ in patches $\left\{\mathcal{P}_{i}\right\}$, cutting up the interface zone in macroelements, each containing a "sufficient" amount of basis functions to construct a patch function $\varphi_{i}>0$ associated to each $P_{i}$ that is zero on the interior patch boundary and takes the value 1 on at least one face cut by the interface. The $\mathcal{P}_{i}$ can be constructed so that

- $\exists c_{1}, c_{2}>0$ such that $c_{1} h \leq \operatorname{diam}\left(P_{i}\right) \leq c_{2} h$. 
- $\exists c_{1}, c_{2}>0 c_{1} h \leq \int_{\Gamma \cap P_{i}} \varphi_{i} \mathrm{~d} s \leq c_{2} h$.

- $\exists c_{1}, c_{2}>0 c_{1} h^{-1} \leq\left|\nabla \varphi_{i}\right| \leq c_{2} h^{-1}$.

We want to prove that we can construct an interpolant $\pi_{h}:\left[H^{1}(\Omega)\right]^{2} \rightarrow V^{h}$ such that

$$
\left\|\boldsymbol{v}-\pi_{h} \boldsymbol{v}\right\|_{0, \Omega}+h\left\|\nabla\left(\boldsymbol{v}-\pi_{h} \boldsymbol{v}\right)\right\|_{0, \Omega_{1} \cup \Omega_{2}} \leq \operatorname{ch}\|\nabla \boldsymbol{v}\|_{0, \Omega}
$$

and

$$
\int_{\Gamma \cap P_{i}}\left(\boldsymbol{v}-\pi_{h}^{k} \boldsymbol{v}\right) \mathrm{d} s=0, \text { for } k=1,2
$$

for all $\mathcal{P}_{i}$.

The idea is to start from the global Clément interpolant of $\boldsymbol{v}$ and then construct a correction using the space $\Phi=\left\{\varphi_{i}\right\}$ so that the condition (22) is satisfied. Let $I_{h} \boldsymbol{v} \in\left[C^{0}(\bar{\Omega})\right]^{2}$ denote the global Clément interpolant of $\boldsymbol{v}$. In each patch $\mathcal{P}_{i}$ there is exactly one (vector valued) degree of freedom $\boldsymbol{y}_{i}$ associated to the basis function $\varphi_{i}$. We know fix the $\boldsymbol{y}_{i}$ by requiring

$$
\int_{\Gamma \cap \mathcal{P}_{i}} \boldsymbol{\varphi}_{i} \boldsymbol{y}_{i} \mathrm{~d} s=\int_{\Gamma \cap \mathcal{P}_{i}}\left(\boldsymbol{v}-I_{h} \boldsymbol{v}\right) \mathrm{d} s .
$$

where

$$
\boldsymbol{\varphi}=\left[\begin{array}{cc}
\varphi_{i} & 0 \\
0 & \varphi_{i}
\end{array}\right]
$$

Finally we set $\pi_{h}^{1} \boldsymbol{v}=\left.I_{h} \boldsymbol{v}\right|_{\mathcal{T}_{1}}+\sum_{i} \boldsymbol{\varphi}_{i} \boldsymbol{y}_{i}$. Clearly, by construction (22) holds. The approximability is a consequence of the stability of the projection. Since $\pi_{h}^{1} \boldsymbol{v}=I_{h} \boldsymbol{v}$, except in the interface zone, we only need to prove stability for the interpolant in $\mathcal{T}_{\Gamma}$. This is where the position of the interface could upset things. The fact that we have chosen the patches to include the neighbours seems to work around this problem. Consider, for some $K \in \mathcal{P}_{i}$

$$
\begin{aligned}
\left\|\nabla \pi_{h}^{1} \boldsymbol{v}\right\|_{K, 0} & \leq\left\|\nabla I_{h} \boldsymbol{v}\right\|_{K \cap \Omega_{1}, 0}+\left\|\nabla\left(\boldsymbol{\varphi}_{i} \boldsymbol{y}_{i}\right)\right\|_{K \cap \Omega_{1}, 0} \\
& \leq c\|\nabla \boldsymbol{v}\|_{K, 0}+c h^{-1}\left|\boldsymbol{y}_{i}\right| \operatorname{meas}(K)^{\frac{1}{2}} \\
& \leq c\left(\|\nabla \boldsymbol{v}\|_{K, 0}+\left|\boldsymbol{y}_{i}\right|\right) .
\end{aligned}
$$

It remains to show that $\left|\boldsymbol{y}_{i}\right|$ is bounded by the norm of $\nabla \boldsymbol{v}$ over some patch of diameter $h$. This follows by using the definition, a Cauchy-Schwarz inequality, 
a trace inequality and the interpolation properties of the Clement interpolant:

$$
\begin{aligned}
\left|\boldsymbol{y}_{i}\right| & \leq\left|\frac{\int_{\Gamma \cap \mathcal{P}_{i}}\left(\boldsymbol{v}-I_{h} \boldsymbol{v}\right) \mathrm{d} s}{\int_{\Gamma \cap \mathcal{P}_{i}}\left(\boldsymbol{\varphi}_{i}: \boldsymbol{\varphi}_{i}\right)^{1 / 2} \mathrm{~d} s}\right| \leq c h^{-1}\left|\int_{\Gamma \cap \mathcal{P}_{i}}\left(\boldsymbol{v}-I_{h} \boldsymbol{v}\right) \mathrm{d} s\right| \\
& \leq c h^{-\frac{1}{2}}\left\|\boldsymbol{v}-I_{h} \boldsymbol{v}\right\|_{0, \Gamma \cap \mathcal{P}_{i}} \\
& \leq c\left(h^{-1}\left\|\boldsymbol{v}-I_{h} \boldsymbol{v}\right\|_{0, \mathcal{P}_{i}}+\left\|\nabla\left(\boldsymbol{v}-I_{h} \boldsymbol{v}\right)\right\|_{0, \mathcal{P}_{i}}\right) \leq c\|\nabla \boldsymbol{v}\|_{0, \Delta_{\mathcal{P}_{i}}},
\end{aligned}
$$

where $\Delta_{\mathcal{P}_{i}}$ denotes the patch consisting of the elements in $\mathcal{P}_{i}$ and those sharing a node with an element in $\mathcal{P}_{i}$. In all interior elements the $H^{1}$-stability is a consequence of the properties of the Clément-interpolant.

The approximability in $L^{2}$ is now easily obtained, noting that for $K \in \mathcal{P}_{i}$

$$
\left\|\boldsymbol{v}-\pi_{h}^{1} \boldsymbol{v}\right\|_{0, K} \leq\left\|\boldsymbol{v}-I_{h} \boldsymbol{v}\right\|_{0, K}+\left\|\boldsymbol{\varphi}_{i} \boldsymbol{y}_{i}\right\|_{0, K} \leq\left\|\boldsymbol{v}-I_{h} \boldsymbol{v}\right\|_{0, K}+c h_{K}\left|\boldsymbol{y}_{i}\right|
$$

and applying the previous result. Using arguments similar to that of Section 4 one may readily prove the stability estimate

$$
\left\|\pi_{h} \boldsymbol{v}\right\| \leq c\|\boldsymbol{v}\|_{1, \Omega}, \quad \forall \boldsymbol{v} \in\left[H^{1}(\Omega)\right]^{2} .
$$

We will now use the proposed interpolant to prove the inf-sup condition for our formulation. Let us start with a preliminary lemma

Lemma 7 Let $v \in\left[H^{1}(\Omega)\right]^{2}$; then there holds, for $i=1,2$,

$$
\left|\left(\llbracket p_{h} \rrbracket, \boldsymbol{n} \cdot\left(\boldsymbol{v}-\pi_{h}^{i} \boldsymbol{v}\right)\right)_{\Gamma}\right| \leq c J\left(p_{h}, p_{h}\right)^{\frac{1}{2}}\left\|\mu_{\max }^{\frac{1}{2}} \nabla \boldsymbol{v}\right\|_{0, \Omega}
$$

PROOF. Using the orthogonality on the patches $\mathcal{P}_{k}$, we may write, with $\xi_{k} \in \mathbb{R}$

$$
\begin{aligned}
\left|\left(\llbracket p_{h} \rrbracket, \boldsymbol{n} \cdot\left(\boldsymbol{v}-\pi_{h}^{i} \boldsymbol{v}\right)\right)_{\Gamma}\right| & \leq \sum_{k}\left|\left(\llbracket p_{h} \rrbracket, \boldsymbol{n} \cdot\left(\boldsymbol{v}-\pi_{h}^{i} \boldsymbol{v}\right)\right)_{\mathcal{P}_{k} \cap \Gamma}\right| \\
& \leq \sum_{k}\left\|\llbracket p_{h} \rrbracket-\xi_{k}\right\|_{0, \mathcal{P}_{k} \cap \Gamma}\left\|\boldsymbol{v}-\pi_{h}^{i} \boldsymbol{v}\right\|_{0, \mathcal{P}_{k} \cap \Gamma} \\
& \leq C \sum_{k}\left\|\llbracket p_{h} \rrbracket-\xi_{k}\right\|_{0, \mathcal{P}_{k} \cap \Gamma} h^{\frac{1}{2}}\|\nabla \boldsymbol{v}\|_{0, \mathcal{P}_{i}} \\
& \leq C\left(h \sum_{k}\left\|\llbracket p_{h} \rrbracket-\xi_{k}\right\|_{0, \mathcal{P}_{k} \cap \Gamma}^{2}\right)^{\frac{1}{2}}\|\nabla \boldsymbol{v}\|_{0, \Omega}
\end{aligned}
$$


We now choose $\xi_{k}$ as the arithmetic mean of all the jumps in the patch $\mathcal{P}_{k}$,

$$
\xi_{k}=\left.\frac{1}{N_{k}} \sum_{K \in \mathcal{P}_{k}: K \cap \Gamma \neq \emptyset} \llbracket p_{h} \rrbracket\right|_{K \cap \Gamma}
$$

where $N_{k}$ denotes the number of triangles in $\mathcal{P}_{k} \cap \mathcal{T}_{\Gamma}$. It then follows, since $N_{k}$ is bounded from above by a moderate constant, uniformly in $h$ and $k$ and using the relation $(a-b)^{2} \leq 2 a^{2}+2 b^{2}$, that for each $K \in \mathcal{P}_{k}$ such that $K \cap \Gamma \neq \emptyset$

$$
\begin{aligned}
\left\|\llbracket p_{h} \rrbracket-\xi_{k}\right\|_{K \cap \Gamma}^{2} \leq & 2\left\|N_{k}^{-1} \sum_{K^{\prime} \in \mathcal{K}^{\prime}}\left(\left.p_{1, h}\right|_{K}-\left.p_{1, h}\right|_{K^{\prime}}\right)\right\|_{0, K \cap \Gamma}^{2} \\
& +2\left\|N_{k}^{-1} \sum_{K^{\prime} \in \mathcal{K}^{\prime}}\left(\left.p_{2, h}\right|_{K}-\left.p_{2, h}\right|_{K^{\prime}}\right)\right\|_{0, K \cap \Gamma}^{2} \\
\leq & C\left(\sum_{F \in \mathcal{E}_{k}} \int_{F}\left[p_{1, h}\right]^{2} \mathrm{~d} s+\int_{F}\left[p_{2, h}\right]^{2} \mathrm{~d} s\right)
\end{aligned}
$$

where $\mathcal{K}^{\prime}:=\left\{K^{\prime} \in \mathcal{P}_{i}: K^{\prime} \cap \Gamma \neq \emptyset ; K^{\prime} \neq K\right\}, \mathcal{E}_{k}:=\left\{F \in \mathcal{P}_{k}: F \cap \Gamma \neq \emptyset\right\}$ and where the constant $C$ depends only on the $N_{k}$. We conclude by summing over all the patches $\mathcal{P}_{i}$.

We will now prove the main result of this section:

Theorem 8 Let $\left(\boldsymbol{u}_{h}, p_{h}\right) \in V_{h} \times Q_{h}$. Then

$$
c_{s} \mid\left\|\left(\boldsymbol{u}_{h}, p_{h}\right)\right\|_{h} \leq \sup _{\left(\boldsymbol{w}_{h}, q_{h}\right) \in V_{h} \times Q_{h}} \frac{B_{h}\left[\left(\boldsymbol{u}_{h}, p_{h}\right),\left(\boldsymbol{v}_{h}, q_{h}\right)\right]+J\left(p_{h}, q_{h}\right)}{\left\|\mid\left(\boldsymbol{v}_{h}, q_{h}\right)\right\|_{h}} .
$$

PROOF. We start by choosing $\left(\boldsymbol{v}_{h}, q_{h}\right)=\left(\boldsymbol{u}_{h}, p_{h}\right)$ to obtain

$$
B_{h}\left[\left(\boldsymbol{u}_{h}, p_{h}\right),\left(\boldsymbol{u}_{h}, p_{h}\right)\right]+J\left(p_{h}, p_{h}\right)=a_{h}\left(\boldsymbol{u}_{h}, \boldsymbol{u}_{h}\right)+J\left(p_{h}, p_{h}\right) .
$$

We will need a version of Korn's inequality for functions in $V_{h}$. From [3] (eq. (1.19)), using $V_{h} \subset\left[H^{1}\left(\Omega_{1} \cup \Omega_{2}\right)\right]^{d}$ and $\left.\boldsymbol{v}\right|_{\partial \Omega}=0$ for $\boldsymbol{v} \in V_{h}$, we have:

$$
\|\boldsymbol{\varepsilon}(\boldsymbol{v})\|_{0, \Omega_{1} \cup \Omega_{2}}^{2}+\|[\boldsymbol{v}]\|_{1 / 2, h, \Gamma}^{2} \geq C|\boldsymbol{v}|_{1, \Omega_{1} \cup \Omega_{2}}^{2} \quad \forall v \in V_{h},
$$

while from [2], using $\left.\boldsymbol{v}\right|_{\partial \Omega}=0,\left.\boldsymbol{v}\right|_{\Gamma_{K}} \in L^{2}\left(\Gamma_{K}\right)$ for $\boldsymbol{v} \in V_{h}$ and a standard error estimate, we have

$$
|\boldsymbol{v}|_{1, \Omega_{1} \cup \Omega_{2}}^{2}+\|[\boldsymbol{v}]\|_{1 / 2, h, \Gamma}^{2} \geq C\|\boldsymbol{v}\|_{0, \Omega}^{2} \quad \forall \boldsymbol{v} \in V_{h}
$$

and thus

$$
\|\boldsymbol{\varepsilon}(\boldsymbol{v})\|_{0, \Omega_{1} \cup \Omega_{2}}^{2}+\|[\boldsymbol{v}]\|_{1 / 2, h, \Gamma}^{2} \geq C_{K}\|\boldsymbol{v}\|_{1, \Omega_{1} \cup \Omega_{2}}^{2} \quad \forall \boldsymbol{v} \in V_{h}
$$


See also the proof on discrete spaces of [7]. Applying now (25), (13) and Lemma 6 , we get

$$
\begin{aligned}
& a_{h}\left(\boldsymbol{u}_{h}, \boldsymbol{u}_{h}\right)+J\left(p_{h}, p_{h}\right) \\
& \geq\left\|\mu^{1 / 2} \boldsymbol{\varepsilon}\left(\boldsymbol{u}_{h}\right)\right\|_{0, \Omega_{1} \cup \Omega_{2}}^{2}-2\left\|\left\{2 \mu \boldsymbol{n} \cdot \boldsymbol{\varepsilon}\left(\boldsymbol{u}_{h}\right)\right\}\right\|_{-1 / 2, h, \Gamma}\left\|\left[\boldsymbol{u}_{h}\right]\right\|_{1 / 2, h, \Gamma} \\
& \quad+\gamma_{u}\left\|\mu_{\max }^{1 / 2}\left[\boldsymbol{u}_{h}\right]\right\|_{1 / 2, h, \Gamma}^{2}+\sum_{i=1}^{2} \sum_{F \in \mathcal{F}_{i}} \frac{\gamma_{i}}{\mu_{i}} h_{K}\left\|\left[p_{i, h}\right]\right\|_{0, F}^{2} \\
& \geq \frac{C_{K}}{2} \mu_{\min }\left\|\boldsymbol{u}_{h}\right\|_{1, \Omega_{1} \cup \Omega_{2}}^{2}+\left(\frac{\mu_{\min }}{2}-\frac{8 C_{I} \mu_{\max }}{\epsilon}\right)\left\|\boldsymbol{\varepsilon}\left(\boldsymbol{u}_{h}\right)\right\|_{0, \Omega_{1} \cup \Omega_{2}}^{2} \\
& +\frac{1}{\epsilon}\left\|\left\{2 \mu \boldsymbol{n} \cdot \boldsymbol{\varepsilon}\left(\boldsymbol{u}_{h}\right)\right\}\right\|_{-1 / 2, h, \Gamma}^{2}+\left(\gamma_{u} \mu_{\max }-\epsilon \mu_{\max }\right)\left\|\left[\boldsymbol{u}_{h}\right]\right\|_{1 / 2, h, \Gamma}^{2} \\
& \quad+\sum_{i=1}^{2} \sum_{F \in \mathcal{F}_{i}} \frac{\gamma_{i}}{\mu_{i}} h_{K}\left\|\left[p_{i, h}\right]\right\|_{0, F}^{2} .
\end{aligned}
$$

Taking $\epsilon=16 C_{I} \frac{\mu_{\max }}{\mu_{\min }}$

$$
c\left\|\boldsymbol{u}_{h}\right\|^{2} \leq B_{h}\left[\left(\boldsymbol{u}_{h}, p_{h}\right),\left(\boldsymbol{u}_{h}, p_{h}\right)\right]+J\left(p_{h}, p_{h}\right)
$$

for $\gamma_{u}>\epsilon$.

By the surjectivity of the divergence operator from $\left[H_{0}^{1}(\Omega)\right]^{2}$ to $L_{0}^{2}(\Omega)$ there exists $\boldsymbol{v}_{p} \in\left[H_{0}^{1}(\Omega)\right]^{2}$ such that $\mu_{\max }^{-1} p_{h}=-\nabla \cdot \boldsymbol{v}_{p}$ and $\left\|\boldsymbol{v}_{p}\right\|_{1, \Omega} \leq c \mu_{\max }^{-1}\left\|p_{h}\right\|_{0, \Omega}$. Taking now $\left(\boldsymbol{w}_{h}, q_{h}\right)=\left(\pi_{h} \boldsymbol{v}_{p}, 0\right)$ where $\pi_{h}$ is the interpolation operator introduced in the previous section we obtain

$$
B_{h}\left[\left(\boldsymbol{u}_{h}, p_{h}\right),\left(\pi_{h} \boldsymbol{v}_{p}, 0\right)\right]=a_{h}\left(u_{h}, \pi_{h} \boldsymbol{v}_{p}\right)+b_{h}\left(p_{h}, \pi_{h} \boldsymbol{v}_{p}-\boldsymbol{v}_{p}\right)+\mu_{\max }^{-1}\left\|p_{h}\right\|_{0, \Omega}^{2} .
$$

Using the Cauchy-Schwarz inequality we have

$$
\begin{aligned}
a_{h}\left(u_{h}, \pi_{h} \boldsymbol{v}_{p}\right) & \geq-\left\|\boldsymbol{u}_{h}\right\|\left|\left\|\pi_{h} \boldsymbol{v}_{p}\right\|\right| \\
& \geq-C\left\|\mid \boldsymbol{u}_{h}\right\| \mu_{\max }^{\frac{1}{2}}\left\|\nabla \boldsymbol{v}_{p}\right\|_{0, \Omega} \geq-C\left\|\boldsymbol{u}_{h}\right\| \mu_{\max }^{-\frac{1}{2}}\left\|p_{h}\right\|_{\Omega} .
\end{aligned}
$$

Using integration by parts in the term $b_{h}\left(p_{h}, \pi_{h} \boldsymbol{v}_{p}-\boldsymbol{v}_{p}\right)$ we have

$$
\begin{aligned}
& b_{h}\left(p_{h}, \pi_{h} \boldsymbol{v}_{p}-\boldsymbol{v}_{p}\right)=\sum_{K \in \mathcal{T}_{h}} \int_{\partial K}\left[p_{h}\right] \boldsymbol{n} \cdot\left(\boldsymbol{v}_{p}-\pi_{h} \boldsymbol{v}_{p}\right) \mathrm{d} s \\
& \quad+\sum_{i} \int_{\mathcal{P}_{i} \cap \Gamma}\left(\llbracket p_{h} \boldsymbol{n} \cdot\left(\boldsymbol{v}_{p}-\pi_{h} \boldsymbol{v}_{p}\right) \rrbracket-\left\{p_{h}\right\} \llbracket \boldsymbol{n} \cdot\left(\boldsymbol{v}_{p}-\pi_{h} \boldsymbol{v}_{p}\right) \rrbracket\right) \mathrm{d} s \\
& =\sum_{K \in \mathcal{T}_{h}} \int_{\partial K}\left[p_{h}\right] \boldsymbol{n} \cdot\left(\boldsymbol{v}_{p}-\pi_{h} \boldsymbol{v}_{p}\right) \mathrm{d} s+\sum_{i} \int_{\mathcal{P}_{i} \cap \Gamma} \llbracket p_{h} \rrbracket \boldsymbol{n} \cdot\left\langle\boldsymbol{v}_{p}-\pi_{h} \boldsymbol{v}_{p}\right\rangle \mathrm{d} s \\
& =I+I I .
\end{aligned}
$$


Treating these term separately we first note that by using the approximation properties of $\pi_{h}$ we have

$$
I \geq-C J\left(p_{h}, p_{h}\right)^{\frac{1}{2}}\left\|\mu_{\max }^{-\frac{1}{2}} p_{h}\right\|_{0, \Omega} .
$$

For the second term we use Lemma 7 which gives

$$
I I \geq-\left|\sum_{i} \int_{\mathcal{P}_{i} \cap \Gamma} \llbracket p_{h} \rrbracket \boldsymbol{n} \cdot\left\langle\boldsymbol{v}_{p}-\pi_{h} \boldsymbol{v}_{p}\right\rangle \mathrm{d} s\right| \geq-C J\left(p_{h}, p_{h}\right)^{\frac{1}{2}}\left\|\mu_{\max }^{-\frac{1}{2}} p_{h}\right\|_{0, \Omega} .
$$

Note that the different weights here are of no importance since the orthogonality property is satisfied on each side separately and since the weights are upper bounded by 1 . We conclude that

$$
B_{h}\left[\left(\boldsymbol{u}_{h}, p_{h}\right),\left(\pi_{h} \boldsymbol{v}_{p}, 0\right)\right] \geq\left\|\mu_{\max }^{-\frac{1}{2}} p_{h}\right\|_{0, \Omega}^{2}-C\left(J\left(p_{h}, p_{h}\right)^{\frac{1}{2}}+\left\|\boldsymbol{u}_{h}\right\| \|\right)\left\|\mu_{\max }^{-\frac{1}{2}} p_{h}\right\|_{0, \Omega}
$$

Using now an arithmethic-geometric inequality we get

$$
B_{h}\left[\left(\boldsymbol{u}_{h}, p_{h}\right),\left(\pi_{h} \boldsymbol{v}_{p}, 0\right)\right] \geq \frac{1}{2}\left\|\mu_{\max }^{-\frac{1}{2}} p_{h}\right\|_{0, \Omega}^{2}-C J\left(p_{h}, p_{h}\right)+C\left\|\boldsymbol{u}_{h}\right\|^{2}
$$

for $\epsilon<C^{-1}$ there holds

$$
c\left\|\mid\left(\boldsymbol{u}_{h}, p_{h}\right)\right\|_{h}^{2} \leq B\left[\left(\boldsymbol{u}_{h}, p_{h}\right),\left(\boldsymbol{u}_{h}+\epsilon \pi_{h} \boldsymbol{v}_{p}, p_{h}\right)\right] .
$$

The claim now follows noting that there exists $c_{s}$ such that

$$
2 c_{s} \mid\left\|\left(\boldsymbol{u}_{h}+\epsilon \pi_{h} \boldsymbol{v}_{p}, p_{h}\right)\right\|_{h} \leq\|\|\left(\boldsymbol{u}_{h}, p_{h}\right) \|_{h},
$$

which is a consequence of the stability of $\pi_{h} \boldsymbol{v}_{p}$.

\subsection{A sketch of the case of one compressible and one incompressible material}

Let assume that $\lambda_{1}$ is bounded but not $\lambda_{2}$. We will then work in the norm

$$
\left\|\left(\boldsymbol{u}_{h}, p_{h}\right)\right\|\left\|_{h}^{2}=\right\| \boldsymbol{u}_{h}\left\|^{2}+\frac{1}{\mu_{\max }}\right\| p_{h}\left\|_{0, \Omega_{2}}^{2}+\lambda_{1}^{-1}\right\| p_{h} \|_{0, \Omega_{1}}^{2}+J\left(p_{h}, p_{h}\right) .
$$

Note that coercivity now gives us

$$
\left\|\boldsymbol{u}_{h}\right\|^{2}+\lambda_{1}^{-1}\left\|p_{h}\right\|_{0, \Omega_{1}}^{2}+J\left(p_{h}, p_{h}\right) \leq B_{h}\left[\left(u_{h}, p_{h}\right),\left(v_{h}, q_{h}\right)\right]+J\left(p_{h}, q_{h}\right) .
$$

However the pressure is no longer in $L_{0}^{2}(\Omega)$. On the other hand we can repeat the above analysis using $v_{p}$ such that $\nabla \cdot v_{p}=\mu_{\max }^{-1}\left(p_{h}-\bar{p}_{h}\right)$, where $\bar{p}_{h}$ denotes the average of $p_{h}$ over $\Omega$. Clearly

$$
\mu_{\max }^{-1}\left\|p_{h}-\bar{p}_{h}\right\|_{0, \Omega}^{2}=\left(p_{h}, \nabla \cdot v_{p}\right)_{\Omega}
$$


and we proceed as before in order to obtain a bound of the quantity:

$$
\lambda_{1}^{-1}\left\|p_{1}\right\|_{0, \Omega_{1}}^{2}+\mu_{\max }^{-1}\left\|p_{h}-\bar{p}_{h}\right\|_{0, \Omega}^{2} .
$$

Now we stress that by the decomposition $p_{i, h}=\left(p_{i, h}-\bar{p}_{i, h}\right)+\bar{p}_{i, h}$ and the orthogonality $\left(\left(p_{i, h}-\bar{p}_{i, h}\right), \bar{p}_{i, h}-\bar{p}_{h}\right)_{\Omega_{i}}=0$ there holds

$\left\|p_{h}-\bar{p}_{h}\right\|_{0, \Omega}^{2}=\left\|p_{2, h}-\bar{p}_{2, h}\right\|_{0, \Omega_{2}}^{2}+\left\|p_{1, h}-\bar{p}_{1, h}\right\|_{0, \Omega_{1}}^{2}+\left\|\bar{p}_{h}-\bar{p}_{1, h}\right\|_{0, \Omega_{1}}^{2}+\left\|\bar{p}_{h}-\bar{p}_{2, h}\right\|_{0, \Omega_{2}}^{2}$.

On the other hand observing that for $i=1,2$

$$
\left\|p_{i, h}\right\|_{0, \Omega_{i}}^{2}=\left\|\bar{p}_{i, h}\right\|_{0, \Omega_{i}}^{2}+\left\|p_{i, h}-\bar{p}_{i, h}\right\|_{0, \Omega_{i}}^{2}
$$

it follows that we only need to bound $\left\|\bar{p}_{2, h}\right\|_{0, \Omega_{2}}^{2}$. Observing that

$$
\begin{aligned}
\left\|\bar{p}_{2, h}\right\|_{0, \Omega_{2}} & \leq\left\|\bar{p}_{h}\right\|_{0, \Omega_{2}}+\left\|\bar{p}_{h}-\bar{p}_{2, h}\right\|_{0, \Omega_{2}} \\
& \leq\left\|\bar{p}_{1, h}\right\|_{0, \Omega_{2}}+\left\|\bar{p}_{h}-\bar{p}_{1, h}\right\|_{0, \Omega_{2}}+\left\|\bar{p}_{h}-\bar{p}_{2, h}\right\|_{0, \Omega_{2}} \\
& \leq\left(\operatorname{meas}\left(\Omega_{2}\right) / \operatorname{meas}\left(\Omega_{1}\right)\right)^{\frac{1}{2}}\left(\left\|\bar{p}_{1, h}\right\|_{0, \Omega_{1}}+\left\|\bar{p}_{h}-\bar{p}_{1, h}\right\|_{0, \Omega_{1}}\right)+\left\|\bar{p}_{h}-\bar{p}_{2, h}\right\|_{0, \Omega_{2}},
\end{aligned}
$$

the required bound now follows from (29), since we have inf-sup control of the right hand.

Note that the constant still degenerates for large $\lambda_{1}$. This reflects the instability of the problem due to the average value of $p_{h}$ that has to be fixed by the small $L^{2}$-coercivity. The same holds for the case where $\lambda_{1}$ is moderate on a very small subdomain $\Omega_{1}$. The quantities $\mu_{\max }^{-1}\left\|p_{1, h}-\bar{p}_{1, h}\right\|_{0, \Omega_{1}}^{2}, \mu_{\max }^{-1}\left\|p_{2, h}-\bar{p}_{2, h}\right\|_{0, \Omega_{2}}^{2}$ and $\mu_{\max }^{-1}\left\|p_{h}-\bar{p}_{h}\right\|_{0, \Omega}^{2}$ however can be controlled independently of $\lambda_{1}$.

\section{A priori error estimates}

Proposition 9 Assume that the solution $(\boldsymbol{u}, p)$ to problem (2) resides in $\left[H^{2}\left(\Omega_{1} \cup \Omega_{2}\right) \cap H^{1}(\Omega)\right]^{2} \times H^{1}\left(\Omega_{1} \cup \Omega_{2}\right) \cap L_{0}^{2}(\Omega)$; then the finite element solution (10) satisfies the error estimate

$$
\left\|\left(\boldsymbol{u}-\boldsymbol{u}_{h}, p-p_{h}\right)\right\| \leq c_{\mu} h\left(\|\boldsymbol{u}\|_{2, \Omega_{1} \cup \Omega_{2}}+\|p\|_{1, \Omega_{1} \cup \Omega_{2}}\right)
$$

where $c_{\mu}$ is independent of $h$ but not of the ratio $\left(\frac{\mu_{\max }}{\mu_{\min }}\right)$.

PROOF. In view of Theorem 3 we only need to show the inequality for $\left\|\left(I_{h}^{*} \boldsymbol{u}-\boldsymbol{u}_{h}, C_{h}^{*} p-p_{h}\right)\right\|$. First we set $\boldsymbol{\eta}_{h}=\boldsymbol{u}_{h}-I_{h}^{*} \boldsymbol{u}$ and $\zeta_{h}=p_{h}-C_{h}^{*} p$ and note that

$$
\left\|\left|\left(\boldsymbol{u}-\boldsymbol{u}_{h}, p-p_{h}\right)\|\mid \leq\|\left(\boldsymbol{u}-I_{h}^{*} \boldsymbol{u}, p-C_{h}^{*} p\right)\|+\|\left(\boldsymbol{\eta}_{h}, \zeta_{h}\right) \|_{h} .\right.\right.
$$


By Theorem 8 and using Galerkin orthogonality we obtain,

$$
\begin{aligned}
\left\|\left(\boldsymbol{\eta}_{h}, \zeta_{h}\right)\right\|_{h} & \leq \frac{1}{c_{s}} \sup _{\left(\boldsymbol{v}_{h}, q_{h}\right) \in V_{h} \times Q_{h}} \frac{B_{h}\left[\left(\boldsymbol{\eta}_{h}, \zeta_{h}\right),\left(\boldsymbol{v}_{h}, q_{h}\right)\right]+J\left(\zeta_{h}, q_{h}\right)}{\left\|\left(\boldsymbol{v}_{h}, q_{h}\right)\right\|_{h}} \\
& \leq \frac{1}{c_{s}} \sup _{\left(\boldsymbol{v}_{h}, q_{h}\right) \in V_{h} \times Q_{h}} \frac{B_{h}\left[\left(\boldsymbol{u}-I_{h}^{*} \boldsymbol{u}, p-C_{h}^{*} p\right),\left(\boldsymbol{v}_{h}, q_{h}\right)\right]-J\left(C_{h}^{*} p, q_{h}\right)}{\left\|\left(\boldsymbol{v}_{h}, q_{h}\right)\right\|_{h}} .
\end{aligned}
$$

It remains to use interpolation estimates to bound the terms on the right hand side. We will make repeated use of inequality (13), Theorem 3 and Lemma 4 below. Treating $B_{h}\left[\left(\boldsymbol{u}-I_{h}^{*} \boldsymbol{u}, p-C_{h}^{*} p\right),\left(\boldsymbol{v}_{h}, q_{h}\right)\right]$ termwise we obtain

$$
\begin{aligned}
& a\left(\boldsymbol{u}-I_{h}^{*} \boldsymbol{u}, \boldsymbol{v}_{h}\right)=\left(2 \mu \boldsymbol{\varepsilon}\left(\boldsymbol{u}-I_{h}^{*} \boldsymbol{u}\right), \boldsymbol{\varepsilon}\left(\boldsymbol{v}_{h}\right)\right)_{0, \Omega_{1} \cup \Omega_{2}}-\left(\left\{2 \mu \boldsymbol{\varepsilon}\left(\boldsymbol{u}-I_{h}^{*} \boldsymbol{u}\right)\right\}, \llbracket \boldsymbol{v}_{h} \rrbracket\right)_{\Gamma} \\
& -\left(\left\{2 \mu \varepsilon\left(\boldsymbol{v}_{h}\right)\right\}, \llbracket \boldsymbol{u}-I_{h}^{*} \boldsymbol{u} \rrbracket\right)_{\Gamma}+\left(\gamma_{u} h^{-1} \llbracket \boldsymbol{u}-I_{h}^{*} \boldsymbol{u} \rrbracket, \llbracket \boldsymbol{v}_{h} \rrbracket\right)_{\Gamma} \\
& \leq\left\|\boldsymbol{u}-I_{h}^{*} \boldsymbol{u}\right\| \mid\left\|\boldsymbol{v}_{h}\right\| \\
& \leq \operatorname{ch}\|\boldsymbol{u}\|_{2, \Omega_{1} \cup \Omega_{2}}\left\|\left(\boldsymbol{v}_{h}, q_{h}\right)\right\|_{h}, \\
& b\left(p-C_{h}^{*} p, \boldsymbol{v}_{h}\right)=-\left(p-C_{h}^{*} p, \nabla \cdot \boldsymbol{v}_{h}\right)+\left(\left\{p-C_{h}^{*} p\right\},\left[\boldsymbol{v}_{h}\right]\right)_{\Gamma} \\
& \leq\left(\left\|\mu_{\max }\left(p-C_{h}^{*} p\right)\right\|_{0, \Omega_{1} \cup \Omega_{2}}+\left\|\left.h\left(p-C_{h}^{*} p\right)\right|_{\Gamma^{+}}\right\|_{0, \Gamma}+\left\|\left.h\left(p-C_{h}^{*} p\right)\right|_{\Gamma^{-}}\right\|_{0, \Gamma}\right) \\
& \times\left(\frac{\mu_{\max }}{\mu_{\min }}\right)\left\|\left(\boldsymbol{v}_{h}, q_{h}\right)\right\|_{h} \\
& \leq c\left(\frac{\mu_{\max }}{\mu_{\min }}\right) h\|p\|_{1, \Omega_{1} \cup \Omega_{2}}\left\|\left(\boldsymbol{v}_{h}, q_{h}\right)\right\|_{h}, \\
& b\left(q_{h}, \boldsymbol{u}-I_{h}^{*} \boldsymbol{u}\right)=\left(q_{h}, \nabla \cdot\left(\boldsymbol{u}-I_{h}^{*} \boldsymbol{u}\right)\right)+\left(\left\{q_{h}\right\},\left[\boldsymbol{u}-I_{h}^{*} \boldsymbol{u}\right]\right)_{\Gamma} \\
& \leq\left(\frac{\mu_{\max }}{\mu_{\min }}\right)\left\|\left(\boldsymbol{u}-I_{h}^{*} \boldsymbol{u}, 0\right)\right\|\|\quad\|\left(\boldsymbol{v}_{h}, q_{h}\right) \mid \|_{h} \\
& +\frac{1}{\gamma_{u}}\left\|\mid\left(\boldsymbol{u}-I_{h}^{*} \boldsymbol{u}, 0\right)\right\|\|\quad\|\left(\boldsymbol{v}_{h}, q_{h}\right)\|\|_{h} \\
& \leq c\left(\frac{\mu_{\max }}{\mu_{\min }}\right) h\|\boldsymbol{u}\|_{2, \Omega_{1} \cup \Omega_{2}}\left\|\mid\left(\boldsymbol{v}_{h}, q_{h}\right)\right\|_{h},
\end{aligned}
$$

and for the weakly consistent term we have

$$
\begin{aligned}
J\left(C_{h}^{*} p, q_{h}\right) & \leq J_{2}\left(p^{*}-C_{h}^{*} p^{*}, p^{*}-C_{h}^{*} p^{*}\right)^{1 / 2} J_{2}\left(q_{h}, q_{h}\right)^{1 / 2} \\
& \leq c\left(\sum_{K \in \mathcal{T}}\left\|h\left(p^{*}-C_{h}^{*} p^{*}\right)\right\|_{\partial K}\right)^{1 / 2} J\left(q_{h}, q_{h}\right) \\
& \leq c h\left\|\mu^{-\frac{1}{2}} p\right\|_{1, \Omega_{1} \cup \Omega_{2}}\left\|\left(\boldsymbol{v}_{h}, q_{h}\right)\right\| \|_{h}
\end{aligned}
$$


Using the Aubin-Nitsche duality argument we prove the following $L_{2}(\Omega)$ estimate for the velocities.

Proposition 10 Under the same assumptions as Proposition 9 there holds

$$
\left\|\boldsymbol{u}-\boldsymbol{u}_{h}\right\|_{0, \Omega} \leq c_{\mu} h^{2}\left(\|\boldsymbol{u}\|_{2, \Omega_{1} \cup \Omega_{2}}+\|p\|_{1, \Omega_{1} \cup \Omega_{2}}\right)
$$

where $c_{\mu}$ is independent of $h$ but not of the ratio $\left(\frac{\mu_{\max }}{\mu_{\min }}\right)$.

PROOF. Let $(\boldsymbol{w}, r) \in V \times Q$ be the solution of the dual equation

$$
B[(\boldsymbol{v}, q),(\boldsymbol{w}, r)]=(\boldsymbol{\psi}, v)_{0, \Omega} \quad \forall(\boldsymbol{v}, q) \in V \times Q,
$$

and we assume that this dual solution enjoys the additional regularity

$$
\|\boldsymbol{w}\|_{2, \Omega_{1} \cup \Omega_{2}}+\|r\|_{1, \Omega_{1} \cup \Omega_{2}} \leq c\|\boldsymbol{\psi}\|_{0, \Omega} .
$$

Choosing $\boldsymbol{v}=\boldsymbol{u}-\boldsymbol{u}_{h}, q=0$ and $\boldsymbol{\psi}=\boldsymbol{u}-\boldsymbol{u}_{h}$, we may write

$$
\left\|\boldsymbol{u}-\boldsymbol{u}_{h}\right\|_{0, \Omega}^{2}=a_{h}\left(\boldsymbol{u}-\boldsymbol{u}_{h}, \boldsymbol{w}\right)+\left(\nabla \cdot\left(\boldsymbol{u}-\boldsymbol{u}_{h}\right), r\right)_{0, \Omega}
$$

and proceed using Galerkin orthogonality and the Cauchy-Schwartz inequality to obtain

$$
\begin{aligned}
\left\|\boldsymbol{u}-\boldsymbol{u}_{h}\right\|_{0, \Omega_{1} \cup \Omega_{2}}^{2}= & a_{h}\left(\boldsymbol{u}-\boldsymbol{u}_{h}, \boldsymbol{w}-I_{h}^{*} \boldsymbol{w}\right)+\left(\nabla \cdot\left(\boldsymbol{u}-\boldsymbol{u}_{h}\right),\left(r-C_{h}^{*} r\right)\right)_{0, \Omega_{1} \cup \Omega_{2}} \\
& +\left(\nabla \cdot I_{h}^{*} \boldsymbol{w}, p-p_{h}\right)_{0, \Omega_{1} \cup \Omega_{2}}+J\left(-p_{h}, C_{h}^{*} r\right) \\
\leq & \left\|\left(\boldsymbol{u}-\boldsymbol{u}_{h}, 0\right)\right\|\left\|_{h}\right\| \mid\left(\boldsymbol{w}-I_{h}^{*} \boldsymbol{w}, 0\right) \|_{h} \\
& +\left\|\nabla \cdot\left(\boldsymbol{u}-\boldsymbol{u}_{h}\right)\right\|_{0, \Omega_{1} \cup \Omega_{2}}\left\|r-C_{h}^{*} r\right\|_{0, \Omega_{1} \cup \Omega_{2}} \\
& +\left|I_{h}^{*} \boldsymbol{w}-\boldsymbol{w}\right|_{1, \Omega_{1} \cup \Omega_{2}}\left\|p-p_{h}\right\|_{0, \Omega_{1} \cup \Omega_{2}} \\
& +J\left(-p_{h}, C_{h}^{*} r^{*}\right) \\
\leq & c\left(\left\|\left(\boldsymbol{u}-\boldsymbol{u}_{h}, p-p_{h}\right)\right\| \mid+J\left(p_{h}, p_{h}\right)^{\frac{1}{2}}\right) \\
& \times\left(\left\|\left(\boldsymbol{w}-I_{h}^{*} \boldsymbol{w}, r-C_{h}^{*} r\right)\right\|+J\left(C_{h}^{*} r^{*}, C_{h}^{*} r^{*}\right)^{\frac{1}{2}}\right) .
\end{aligned}
$$

As a consequence of Proposition 9, Theorem 3, Lemma 5 and the regularity hypothesis (31) we may conclude that

$$
\begin{aligned}
\left\|\boldsymbol{u}-\boldsymbol{u}_{h}\right\|_{0, \Omega_{1} \cup \Omega_{2}}^{2} & \leq C h\left(\|\boldsymbol{w}\|_{2, \Omega_{1} \cup \Omega_{2}}+\|r\|_{1, \Omega_{1} \cup \Omega_{2}}\right)\left\|\left(\boldsymbol{u}-\boldsymbol{u}_{h}, p-p_{h}\right)\right\|_{h} \\
& \leq C h^{2}\left\|\boldsymbol{u}-\boldsymbol{u}_{h}\right\|_{0, \Omega_{1} \cup \Omega_{2}}\left(\|\boldsymbol{u}\|_{2, \Omega_{1} \cup \Omega_{2}}+\|p\|_{1, \Omega_{1} \cup \Omega_{2}}\right),
\end{aligned}
$$

and the result follows. 


\section{$7 \quad$ Numerical results}

\subsection{An artificial interface in an incompressible medium}

Our first example shows that we have optimal convergence in the case of an artificial interface in an incompressible solid with constant material parameters. The problem, often used in the context of Stokes problem of creeping flow, is posed on the unit square with exact solution given by $\boldsymbol{u}=\left(20 x y^{3}, 5 x^{4}-5 y^{4}\right)$ and $p=60 x^{2} y-20 y 3-5$. Choosing $\gamma_{1,2}=1$ and $\gamma_{\mu}=10$, we obtain the optimal convergence shown in Figure 1, first order for the pressure and second order for the displacements. The position of the artificial interface is shown in Fig. 2 on one of the computational meshes in the sequence.

\subsection{An elastic interface problem with $\nu_{1} \rightarrow 1 / 2$}

We have considered a radially symmetric problem with different material properties in concentric discs around the origin. The inner disc has material parameters $E_{1}, \nu_{1}$, and the outer $E_{2}, \nu_{2}$. At any point, the displacement vector can be written $\boldsymbol{u}=\left(u_{r}, u_{\theta}\right)$, where $u_{r}$ is the radial component of the displacement and $u_{\theta}$ is the circumferential component. The material is subjected to a boundary displacement $\boldsymbol{u}=\mathbf{x}$ (in Cartesian coordinates), and the exact solution to the problem is given by (cf. [10])

$$
\begin{aligned}
& u_{r}(r)=\left\{\begin{array}{l}
\left(\left(1-\frac{b^{2}}{a^{2}}\right) c+\frac{b^{2}}{a^{2}}\right) r, \quad 0 \leq r \leq a, \\
\left(r-\frac{b^{2}}{r}\right) c+\frac{b^{2}}{r}, \quad a \leq r \leq b,
\end{array}\right. \\
& u_{\theta}=0,
\end{aligned}
$$

with

$$
c=\frac{\left(\lambda_{1}+\mu_{1}+\mu_{2}\right) b^{2}}{\left(\lambda_{2}+\mu_{2}\right) a^{2}+\left(\lambda_{1}+\mu_{1}\right)\left(b^{2}-a^{2}\right)+\mu_{2} b^{2}} .
$$

When $\nu_{1}=1 / 2$, the last relation degenerates to $c=1 /\left(b^{2}-a^{2}\right)$. We have set $E_{1}=E_{2}=1, \nu_{2}=0.25$ and set $\nu_{1} \in\{0.3,0.49,0.5\}$. The problem was solved on a quarter of a disc with symmetry boundary conditions on the vertical and horizontal boundaries and with the given boundary condition on the circumference. The stability parameters were chosen as in the previous example.

In Figure 3 we show the convergence in displacements for the different $\nu_{1}$. We obtain second order convergence in all cases, though the error on a given mesh 
increases with $\nu_{1}$. We also show, in Figure 4, an elevation of the length of the displacement vectors and the $p$ variable for $\nu_{1}=0.49$.

\section{References}

[1] R. Becker and P. Hansbo. A simple pressure stabilization method for the Stokes equation. Comm. Numer. Methods Eng.

[2] S. C. Brenner. Poincaré-Friedrichs inequalities for piecewise $H^{1}$ functions. SIAM J. Numer. Anal., 41(1):306-324, 2003.

[3] S. C. Brenner. Korn's inequalities for piecewise $H^{1}$ vector fields. Math. Comp., 73(247):1067-1087, 2004.

[4] F. Brezzi and J. Pitkäranta. On the stabilization of finite element approximations of the Stokes equations. In Efficient solutions of elliptic systems (Kiel, 1984), volume 10 of Notes Numer. Fluid Mech., pages 11-19. Vieweg, Braunschweig, 1984 .

[5] A. Hansbo and P. Hansbo. An unfitted finite element method, based on Nitsche's method, for elliptic interface problems. Comput. Methods Appl. Mech. Engrg., 191(47-48):5537-5552, 2002.

[6] A. Hansbo and P. Hansbo. A finite element method for the simulation of strong and weak discontinuities in solid mechanics. Comput. Methods Appl. Mech. Engrg., 193(33-35):3523-3540, 2004.

[7] P. Hansbo and M. G. Larson. Discontinuous Galerkin methods for incompressible and nearly incompressible elasticity by Nitsche's method. Comput. Methods Appl. Mech. Engrg., 191(17-18):1895-1908, 2002.

[8] D. Leguillon and E. Sánchez-Palencia. Computation of singular solutions in elliptic problems and elasticity. John Wiley $\mathcal{G}$ Sons, Ltd., Chichester; Masson, Paris, 1987. 200 pp.

[9] Y. Shibata and S. Shimizu. On a resolvent estimate of the interface problem for the Stokes system in a bounded domain. J. Differential Equations 191, no. 2, 408-444, 2003.

[10] N. Sukumar, D.L. Chopp, N. Möes, and T. Belytschko, Modeling holes and inclusions by level sets in the extended finite-element method, Comput. Methods Appl. Mech. Engrg., 190 6183-6200, 2001. 


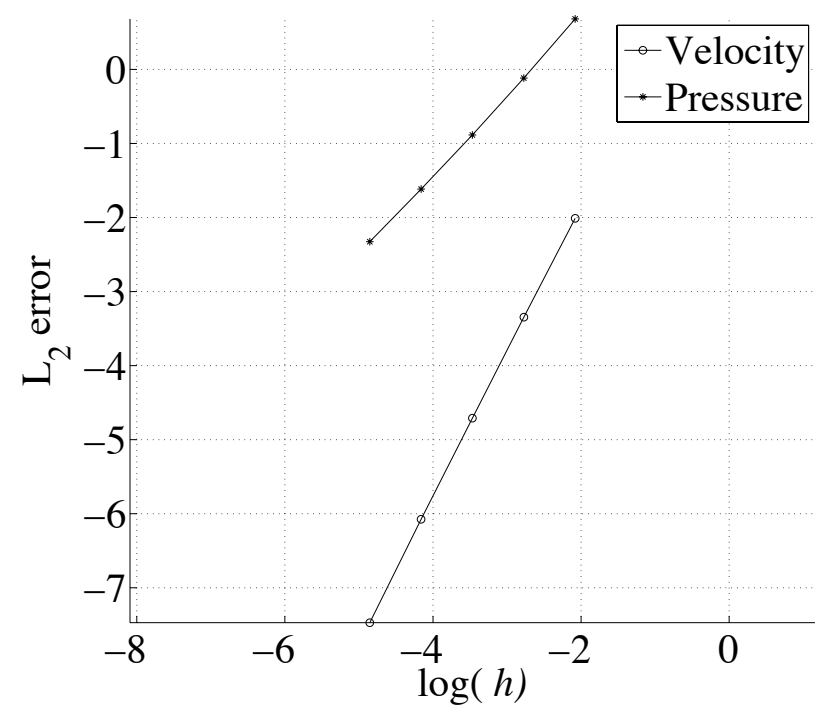

Fig. 1. Convergence for an artificial interface.

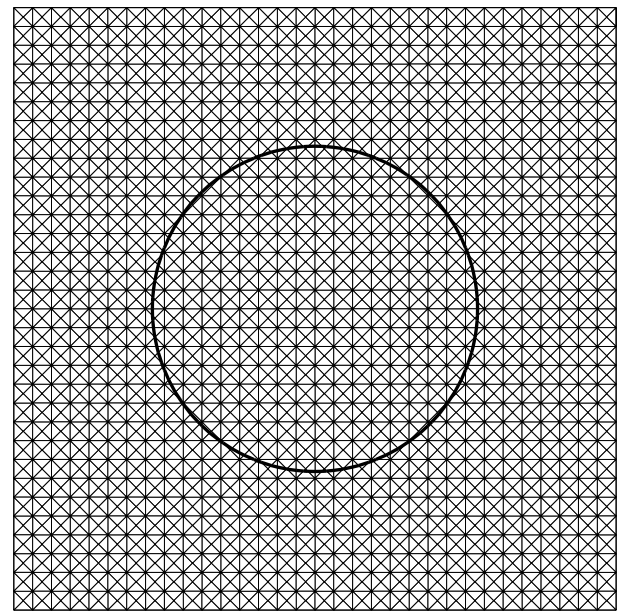

Fig. 2. Position of the artificial interface. 


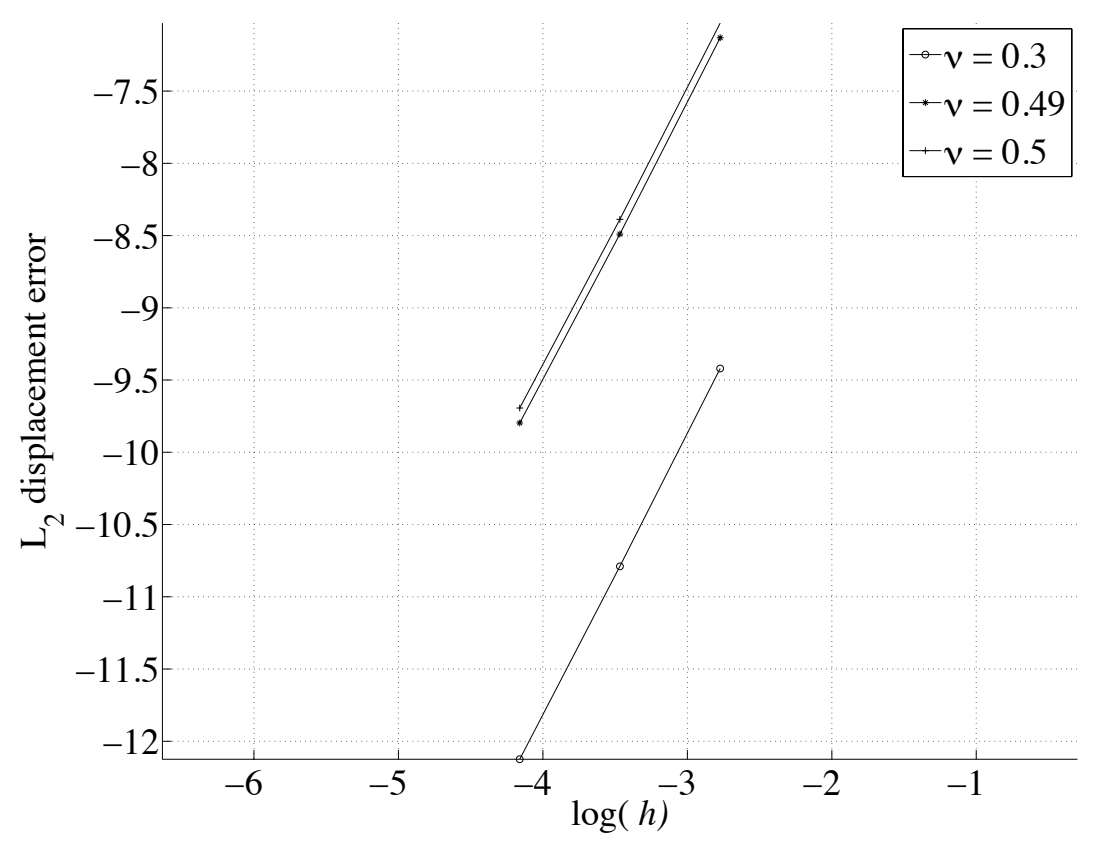

Fig. 3. Convergence for the elasticity problem.
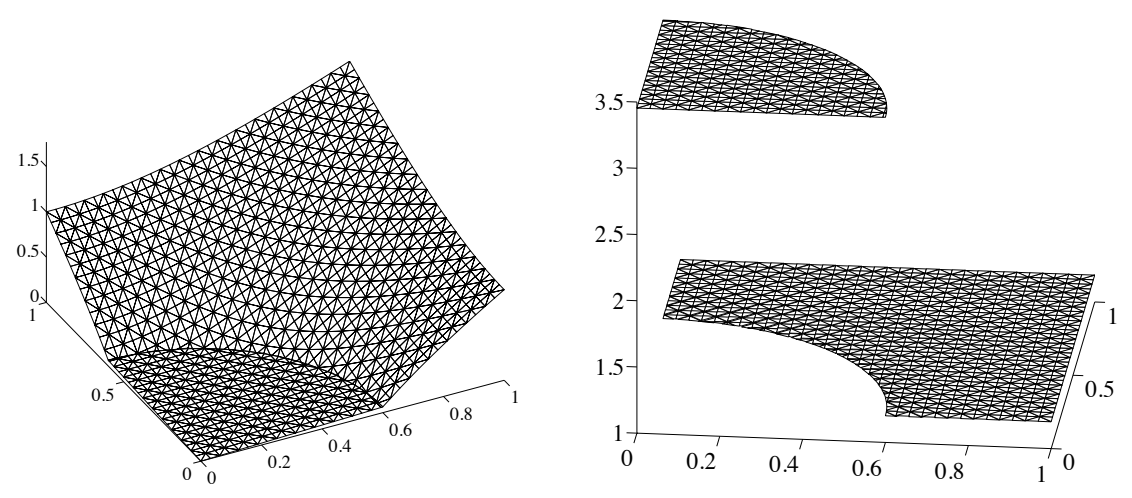

Fig. 4. Elevation of $|u|$ and $p$ for $\nu_{1}=0.49$. 